\title{
Noncontact Optical Fiber Sensor for Measuring the Refractive Index of Liquids
}

\author{
R. Selvas-Aguilar, ${ }^{1}$ A. Castillo-Guzman, ${ }^{1}$ L. Cortez-Gonzalez, ${ }^{1}$ D. Toral-Acosta, ${ }^{1}$ \\ A. Martinez-Rios, ${ }^{2}$ G. Anzueto-Sanchez, ${ }^{3}$ V. M. Duran-Ramirez, ${ }^{4}$ and S. Arroyo-Rivera ${ }^{1}$ \\ ${ }^{1}$ Facultad de Ciencias Físico Matemáticas, Universidad Autónoma de Nuevo Leon, Avenida Universidad S/N, \\ Cd. Universitaria, 66455 San Nicolás de los Garza, NL, Mexico \\ ${ }^{2}$ Centro de Investigaciones en Optica, A.C., Loma del Bosque 115, 37150 Leon, GTO, Mexico \\ ${ }^{3}$ Centro de Investigacion en Ingenieria y Ciencias Aplicadas, Universidad Autónoma del Estado de Morelos, \\ Avenida Universidad No. 1001, Colonia Chamilpa, 62209 Cuernavaca, MOR, Mexico \\ ${ }^{4}$ Centro Universitario de los Lagos, Universidad Autónoma de Guadalajara, Enrique Diaz de Leon 1144, \\ 47460 Lagos de Moreno, JAL, Mexico
}

Correspondence should be addressed to R. Selvas-Aguilar; rselvas@gmail.com

Received 27 November 2015; Accepted 17 February 2016

Academic Editor: Xingwei Wang

Copyright (C) 2016 R. Selvas-Aguilar et al. This is an open access article distributed under the Creative Commons Attribution License, which permits unrestricted use, distribution, and reproduction in any medium, provided the original work is properly cited.

A noncontact optical fiber sensor for measuring the refractive index of transparent liquids is proposed. It operates by calculating the path of a focused laser beam at $635 \mathrm{~nm}$ that travels across the boundaries of a liquid sample. The optical power Fresnel reflections are detected and, subsequently, the refractive index is determined as the ratio between the traveled beam paths when the liquid is deposited versus a reference without the liquid sample. Additionally, a mathematical analysis of the geometrical case is included. The theoretical data from our sensor are in good agreement with the experimental results. The resolution achieved by the sensor is better than $10^{-3}$ RIU.

\section{Introduction}

In recent years, sensor devices have been widely used for physical and chemical quantities measurements in some manufacturing processes and gadgets such as small size and high sensitivity sensors [1]. These kinds of sensors have gained great interest because of their wide demand for implementation in many industrial processes. Such processes require the monitoring of certain properties in liquids; for example, these kinds of sensors are used in fields of study such as the chemicals, food industry, biotech, medical measurement, and environment assessment or in research laboratories.

Some properties of these liquids, such as the refractive index, have been considered as one of the most important parameters used to be monitored. A well-known device typically used for this purpose is the traditional Abbe refractometer [2] or in a similar way one can find setups that employ the ray deviation through a glass-liquid interface to measure the refractive index of transparent liquids [3-5]. Those works offer accurate results, but under the point of view of simplicity and compactness they result rather in being bulky. Notwithstanding, recent advances in optoelectronics have motivated the development of novel fiber-optic refractive index (RI) sensors. Fiber-optic based sensors have, therefore, the same advantages attributable to other sensors plus the advantages owing to their waveguide, their electromagnetic immunity, and their remote sensing capability, among others. Regarding these novel devices, there are a couple of research groups $[6,7]$ who demonstrated that, by using the Fresnel reflections exhibited at the liquid-glass interface of fiber probes, it is possible to obtain a ratio between a reference and a test signal to calculate liquids refractive indices. More recently, another setup also based on a Fresnel reflection added only an optical switch to obtain the signal ratios resulting in an enhanced accuracy for the measurement of RI [8]; additionally, in [9], a time-delayed optical signal through a remote large fiber 


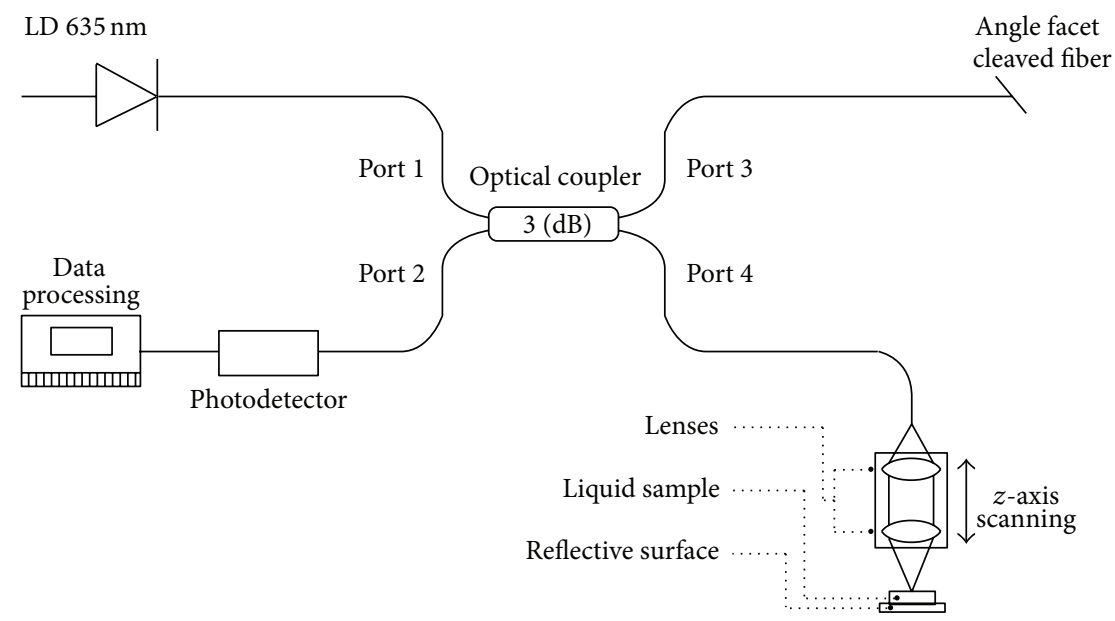

FIgURE 1: Optical fiber refractometer setup.

segment is employed to obtain the same signal ratio in the measurement. A slightly different approach uses a tapered optical fiber $[10,11]$ where the immersion of a fiber-tapered section in the liquid sample produces changes in the evanescent field which allow calculating the RI. Finally in [12], the measurement process of the RI was based on the shift in the interference pattern as a result of a fiber inline micronotch cavity. The results of the above-cited works are important in measurements where the immersion of the fiber probe into the liquid sample is necessary; however it can be a potential drawback when a noncontact measurement is required.

In this paper, a versatile noncontact optical fiber sensor for measuring the refractive index of a transparent liquid is proposed. This setup employs a laser diode at $635 \mathrm{~nm}$ and operates as a function of the launched light into the boundaries of a liquid sample. By means of the back-reflected optical intensities in a photodetector, the RI is consequently calculated as the ratio between the traveled beam paths when liquid is deposited versus a reference without the liquid sample. The system proved to be compact, robust, repeatable, and reliable, and unlike liquid-glass fiber-based sensors, our system operates without the requirement of immersion of the fiber probe into the liquid sample. Interestingly, since the terminal sensor is not required in a degradation process, this sensor guarantees a longer lifetime due to its low maintenance requirements. Furthermore, a mathematical analysis of the geometrical case for the refractive index principle is shown. The theoretical data calculated from our sensor are in good agreement with the experimental results. Refractive indices of the tested liquids were measured at a wavelength of $635 \mathrm{~nm}$ and with a resolution of $10^{-3}$ RIU.

\section{Optical Fiber Refractometer}

Figure 1 shows the experimental setup of the refractometer. It consists of an optical fiber-based setup and a laser light. Attached to a motorized $z$-axis mechanism, they make a scanning sweep along its vertical $z$-axis to get the corresponding retroreflected optical power for every step along the way.
A stabilized laser source at $635 \mathrm{~nm}$ (Thorlabs LPS-635FC) with controlled temperature is fusion-spliced to port 1 of a $3 \mathrm{~dB}$ optical fiber coupler $(2 \times 2$ wideband fiber optical coupler $630 \mathrm{~nm}$ Thorlabs TW630R5F2) as shown in Figure 1. Coupled to fiber end (single mode fiber 600 to $860 \mathrm{~nm}$ Thorlabs SM600) at port 4, a pair of lenses collimates and focuses the laser beam; this arrangement serves as the fiber probe for the sensor. The proposed positioning scheme for the $z$-axis-scanner mechanism is based on a motorized actuator [16] (Thorlabs Z812B) which is set to allow moving the fiber probe backward and forward along all the way of the liquid sample under test. Every position where the focused light is set to reach the boundaries of two different planar media corresponds to a Fresnel reflection which retroreflects the optical power back to the fiber probe. For this reason a fiberpigtailed photodetector (Thorlabs FDSP660) is spliced to port 2 in order to measure the retroreflected optical power.

It is worth mentioning that the fiber in port 3 of the optical coupler has an angle facet cleaved to prevent any feedback of light which in turn could cause a significant error in our measurement.

\section{Mathematical Analysis for Refractive Index Measurement}

Let us now consider that the sample is illuminated by the fiber probe's output laser beam. Tracing the incident and refracted rays at the interface of two different media with refractive indexes $n_{1}$ and $n_{2}$ and the lengths $h_{1}$ and $h_{2}$ in Figure 2, it is possible to write the following geometrical analysis.

Using Snell's law,

$$
n_{1} \sin \theta_{1}=n_{2} \sin \theta_{2} .
$$

Considering the geometry of the angles, we can demonstrate that

$$
n_{2}=n_{1}\left(\frac{1}{r_{1}}\right) r_{2} \text {. }
$$




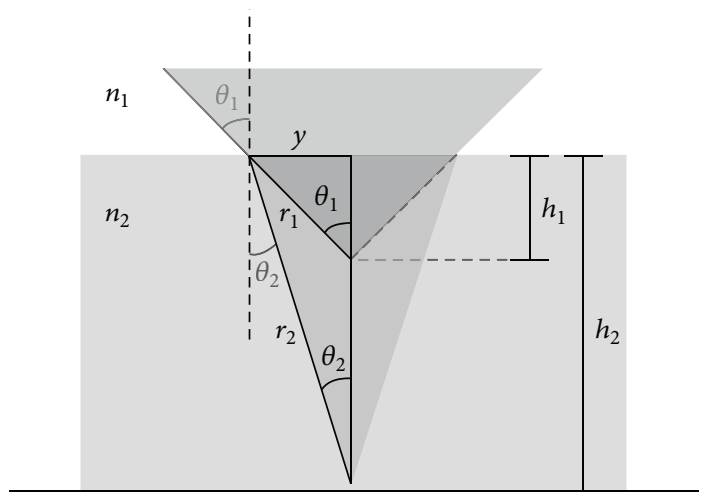

FIGURE 2: Geometrical analysis of refracted light.

Equation (2) can be also stated in terms of the incident angle and the values of $h_{1}$ and $h_{2}$ as follows:

$$
n_{2}=n_{1}\left(\frac{\cos \theta_{1}}{h_{1}}\right) \sqrt{y^{2}+h_{2}^{2}}
$$

Given the fact that $y$ has the same value for both angles, we can express (3) in terms of the incident angle and therefore get the resultant equation for the refractive index of the sample, which can finally be stated as

$$
n_{2}=n_{1}\left(\frac{\cos \theta_{1}}{h_{1}}\right) \sqrt{\left(h_{1}^{2} \tan ^{2} \theta_{1}\right)+h_{2}^{2}}
$$

\section{Measurement Process}

The operation principle of the refractometer is based on the optical path length traveled by a ray of light to reach a mirror target through a sample and without it. For this purpose, it is then necessary to know the thickness of the sample. In general, this can be obtained given the fact that, in every planar interface between the two media, a high intensity Fresnel reflection is exhibited each time such interfaces are reached by the focal point.

Given the experimental setup and the analysis of the mathematical model applicable to describe the refractometer in Section 3, a method must be provided to find the values of $h_{1}$ and $h_{2}$. A better understanding of this can be achieved by considering the details of the whole measurement process.

Regarding Figure 3, if we assume that the motorized mechanism starts moving from the zero-reference position, the laser focal point will sometime reach a reflective interface (i.e., air-liquid, liquid-metallic mirror, or air-metallic mirror interface). Subsequently, the retroreflected light enters back into the fiber probe and it is then guided to the optical fiber coupler where a photodetector will receive the reflected optical power and convert it into a voltage signal. For each $z$ axis position where the focal point reaches one high reflective surface, the voltage signal exhibits a local maximum. Finally, the voltage signal is computer-monitored and plotted for each vertical position.
Once a scanning sweep is performed, it is possible to find the desired $h_{1}$ and $h_{2}$ values. From Figures 2 and 3, it can be confirmed that

$$
h_{1}=\left|L_{3}-L_{2}\right| \text {, }
$$

where $L_{2}$ is the distance from the reference position to the sample surface and $L_{3}$ is the required displacement from the reference to a position such that the focal point of the light has completely penetrated the sample and reached the sample holder's reflective bottom.

Additionally, we can calculate $h_{2}$ as

$$
h_{2}=\left|L_{1}-L_{2}\right| \text {, }
$$

where $L_{1}$ is the distance of the sample holder's bottom with respect to the reference position.

Consequently the measurement process can be done in terms of the values $L_{1}, L_{2}$, and $L_{3}$. In order to complete the process, it is necessary to implement only two steps: (i) without a sample in the sample holder, a scanning sweep must be carried out to obtain $L_{1}$, and (ii) with a layer of sample added in the sample holder, $L_{2}$ and $L_{3}$ must be obtained.

\section{Experimental Results}

To determine the repeatability and accuracy in the experiment, a series of measurements were performed. On the one hand, by choosing randomly two different samples, standard deviation was obtained out of 10 measured values. The results showed an agreement better than $10^{-3}$. See Figure 4 .

On the other hand, in order to verify the accuracy of our device at different sample thickness, some measurements were performed using glycerin. The obtained error remained in a value $<10^{-3}$. See Figure 5. Moreover, the sensor exhibited good performance even when thickness was reduced down to $\sim 1 \mathrm{~mm}$, which corresponds to $10 \%$ of the focal length of the optical system.

Table 1 shows a comparison between the refractive index values obtained for different substance samples measured by our refractometer (patent pending) [17] with respect to the values obtained using a commercial analog Abbe refractometer (model Atago 1T). An additional column shows the 


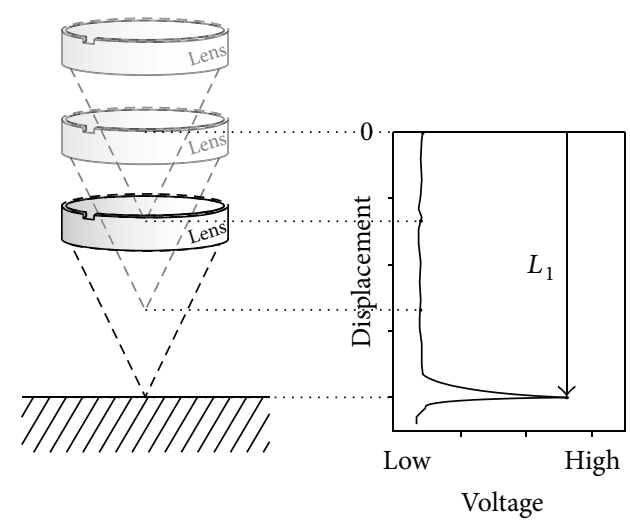

(a)

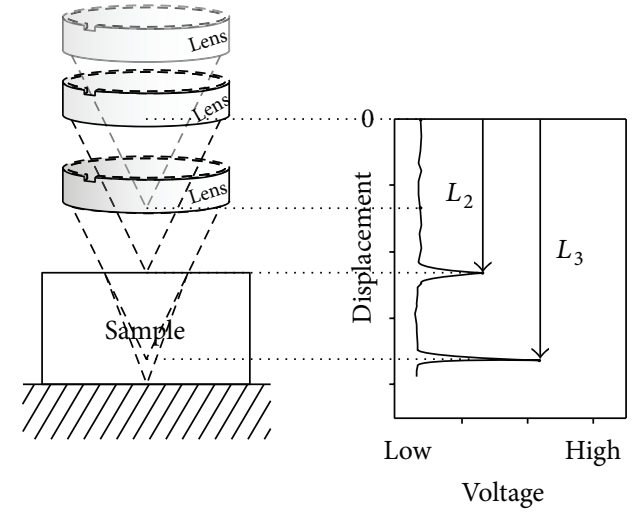

(b)

FIGURE 3: Obtaining process of the refractive index. (a) Measurement implemented with an empty sample holder shows a single reflective surface. (b) Measurement with a liquid sample on the sample holder gives two reflective surfaces.

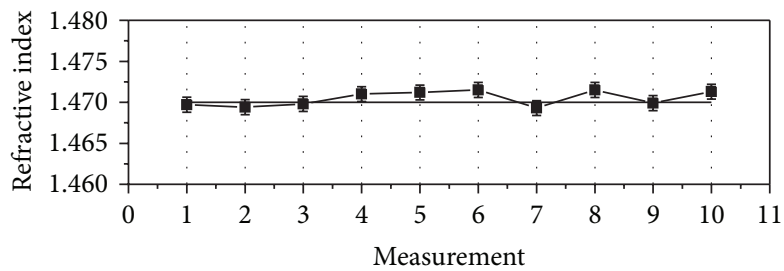

- Glycerin

Error: $9.13 E-4$

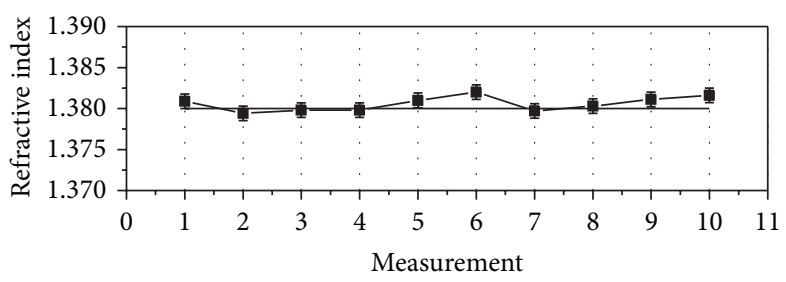

- Isopropyl alcohol

Error: 8.859E - 4

FIGURE 4: Standard deviation in measurements with glycerin and isopropyl alcohol. Straight lines (horizontal) represent reported values. 
TABLE 1: Comparison of results with reported values.

\begin{tabular}{lccc}
\hline Substance & Proposed refractometer $\left(25^{\circ} \mathrm{C}\right)$ & Commercial Abbe refractometer $\left(23.5^{\circ} \mathrm{C}\right)$ & $\begin{array}{c}\text { Reported in literature } \\
{[2,13-15]}\end{array}$ \\
\hline Distilled water & 1.3346 & 1.3344 & $1.3327\left(25^{\circ} \mathrm{C}\right)$ \\
Hydrogen peroxide $(40 \% \mathrm{wt})$ & 1.3579 & 1.3625 & $1.3598\left(25^{\circ} \mathrm{C}\right)$ \\
Glycerin & 1.4757 & 1.4685 & $1.4739\left(20^{\circ} \mathrm{C}\right)$ \\
Isopropyl alcohol & 1.3798 & 1.3819 & $1.378\left(20^{\circ} \mathrm{C}\right)$ \\
\hline
\end{tabular}
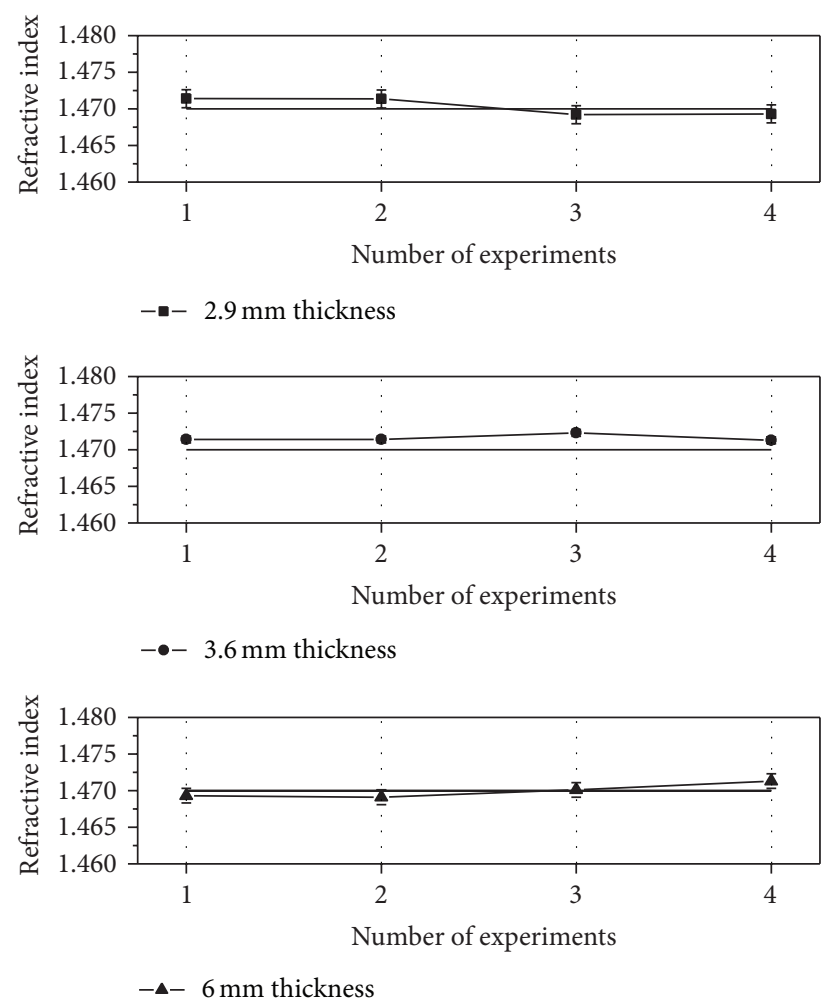

Figure 5: Measurements with different thicknesses of glycerin samples showed an error $<10^{-3}$. Straight lines (horizontal) represent the reported value of glycerin.

corresponding refractive index values of the same substances reported in literature.

It is worth mentioning that each measured refractive index exhibited small deviations with respect to reference values reported. The fact that reported values of refractive index in literature are given for a sodium D-line $(589 \mathrm{~nm})$ must be considered, while in our system a $635 \mathrm{~nm}$ laser wavelength was used. In fact, most measured substances show a decreasing refractive index as the wavelength increases. On the other hand, the optical power of the laser used for the measurements was $0.4 \mathrm{~mW}$, which means that we can discard any thermal issue related to the incident light over the amount of analyzed substance. We registered all the measurements in a labcontrolled temperature, performing the experiment in about 6 seconds. We addressed possible shake issues by attaching the motorized $z$-axis-mechanism to an overweighed plate.

\section{Conclusions}

The mathematical analysis and the obtained experimental results demonstrated that the deviation of the principal ray of the laser beam when it emerges from the sample holder by Fresnel reflection throughout a transparent liquid sample and without it can be determined in terms of the scanning displacements that set the focal point of the focused beam at the boundaries of the liquid sample. As a result, we can determine the refractive index of the liquid with simple measurements without the requirement of physical contact. The measured refractive index values of liquids such as distilled water, hydrogen peroxide, glycerin, and isopropyl alcohol closely agree with the reported values and the measured values using a commercial refractometer. The optical system exhibited a good accuracy for thicknesses as low as $1 \mathrm{~mm}$ under the chosen lenses for the fiber probe. Smaller thickness resulted in an inaccurate calculation of RI.

\section{Competing Interests}

The authors declare that they have no competing interests.

\section{Acknowledgments}

This work was supported in part by the Scientific and Technology Research Support Program (PAICyT), hosted by the Universidad Autónoma de Nuevo Leon (UANL), Projects Estudio y Caracterización de Linternas Fotónicas de Modos Selectivos (MSPLs), IT1047-11, CA354, and IT1087-11, by Secretaría de Educación Pública by Projects PROFOCIE 2015, PRODEP-REDES 103.5/15/11043, and by the PROMEP, hosted by UANL, Project 103.5/13/6444. D. Toral-Acosta was supported by the National Council of Science and Technology (CONACyT) Grant no. 260496. The authors also want to thank to Adriana Liñan Montes, Daniel Ceballos Herrera, Helena de los Reyes, and Jaime Reyes for their valuable comments on the paper.

\section{References}

[1] S. Yio, P. B. B Ruffin, F. T. S. Yu, and S. Yin, Fiber Optic Sensors, Marcel Dekker, New York, NY, USA, 2008.

[2] J. Rheims, J. Köser, and T. Wriedt, "Refractive-index measurements in the near-IR using an Abbe refractometer," Measurement Science and Technology, vol. 8, no. 6, pp. 601-605, 1997.

[3] M. Daimon and A. Masumura, "Measurement of the refractive index of distilled water from the near-infrared region to the 
ultraviolet region," Applied Optics, vol. 46, no. 18, pp. 3811-3820, 2007.

[4] S. Nemoto, "Measurement of the refractive index of liquid using laser beam displacement," Applied Optics, vol. 31, no. 31, pp. 6690-6694, 1992.

[5] F. Docchio, S. Corini, M. Perini, and R. S. Gasana, "A simple and reliable system for measuring the refractive index of liquids using a position-sensitive detector," IEEE Transactions on Instrumentation and Measurement, vol. 44, no. 1, pp. 68-70, 1995.

[6] P. Nath, H. K. Singh, P. Datta, and K. C. Sarma, "All-fiber optic sensor for measurement of liquid refractive index," Sensors and Actuators A: Physical, vol. 148, no. 1, pp. 16-18, 2008.

[7] C.-B. Kim and C. B. Su, "Measurement of the refractive index of liquids at 1.3 and 1.5 micron using a fibre optic Fresnel ratio meter," Measurement Science and Technology, vol. 15, no. 9, pp. 1683-1686, 2004.

[8] W. Xu, X. G. Huang, and J. S. Pan, "Simple fiber-optic refractive index sensor based on fresnel reflection and optical switch," IEEE Sensors Journal, vol. 13, no. 5, pp. 1571-1574, 2013.

[9] J. Yuan, C. Zhao, M. Ye, J. Kang, Z. Zhang, and S. Jin, "A Fresnel reflection-based optical fiber sensor system for remote refractive index measurement using an OTDR," Photonic Sensors, vol. 4, no. 1, pp. 48-52, 2014.

[10] J.-F. Ding, A. P. Zhang, L.-Y. Shao, J.-H. Yan, and S. He, "Fibertaper seeded long-period grating pair as a highly sensitive refractive-index sensor," IEEE Photonics Technology Letters, vol. 17, no. 6, pp. 1247-1249, 2005.

[11] A. Iadicicco, A. Cusano, A. Cutolo, R. Bernini, and M. Giordano, "Thinned fiber Bragg gratings as high sensitivity refractive index sensor," IEEE Photonics Technology Letters, vol. 16, no. 4, pp. 1149-1151, 2004.

[12] T. Wei, Y. Han, Y. Li, H.-L. Tsai, and H. Xiao, “Temperatureinsensitive miniaturized fiber inline Fabry-Perot interferometer for highly sensitive refractive index measurement," Optics Express, vol. 16, no. 8, pp. 5764-5769, 2008.

[13] P. R. Cooper, "Refractive-Index measurements of liquids used in conjunction with optical fibers," Applied Optics, vol. 22, no. 19, pp. 3070-3072, 1983.

[14] E. Moreels, C. de Greef, and R. Finsy, "Laser light refractometer," Applied Optics, vol. 23, no. 17, pp. 3010-3013, 1984.

[15] http://www.refractometer.pl/refraction-datasheet-basic.

[16] http://www.thorlabs.com/newgrouppage9.cfm?objectgroup_id= $1882 \& p n=Z 812 B \# 2497$.

[17] R. Selvas, A. Castillo, L. Cortez, and D. Toral, "Sistema Detector de no contacto de Espesor y Propiedades Ópticas en Materiales y Líquidos Apilados o Crecidos en Multicapas," IMPI Patente no. MX/E/2014/064537, 2014. 


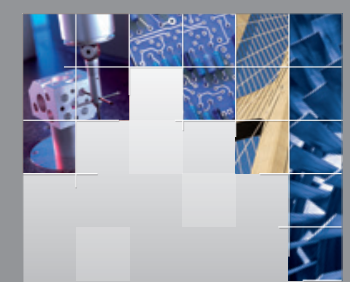

\section{Enfincering}
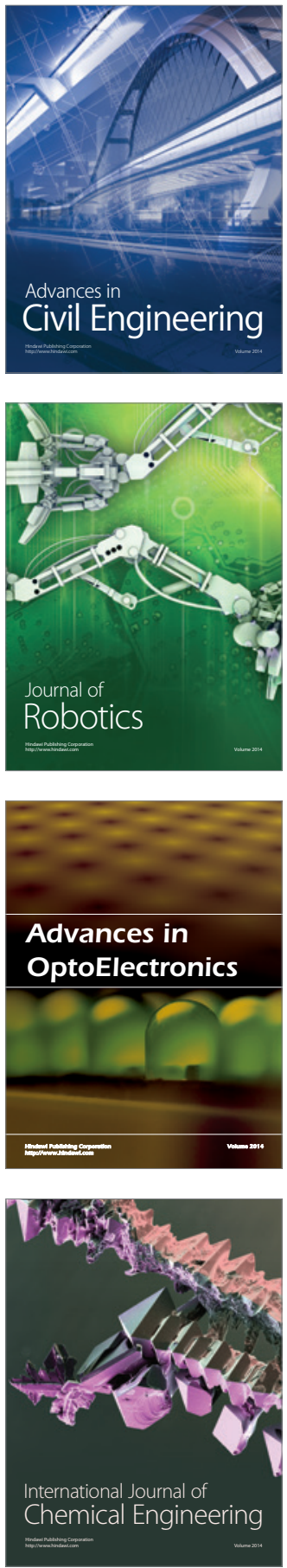

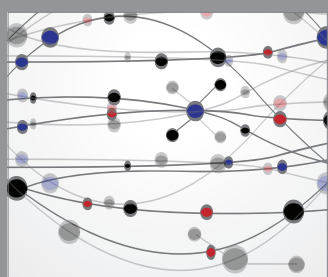

The Scientific World Journal

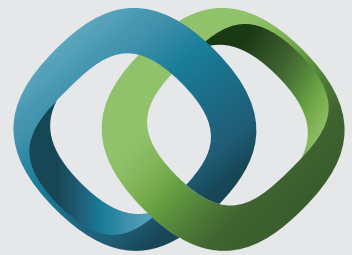

\section{Hindawi}

Submit your manuscripts at

http://www.hindawi.com
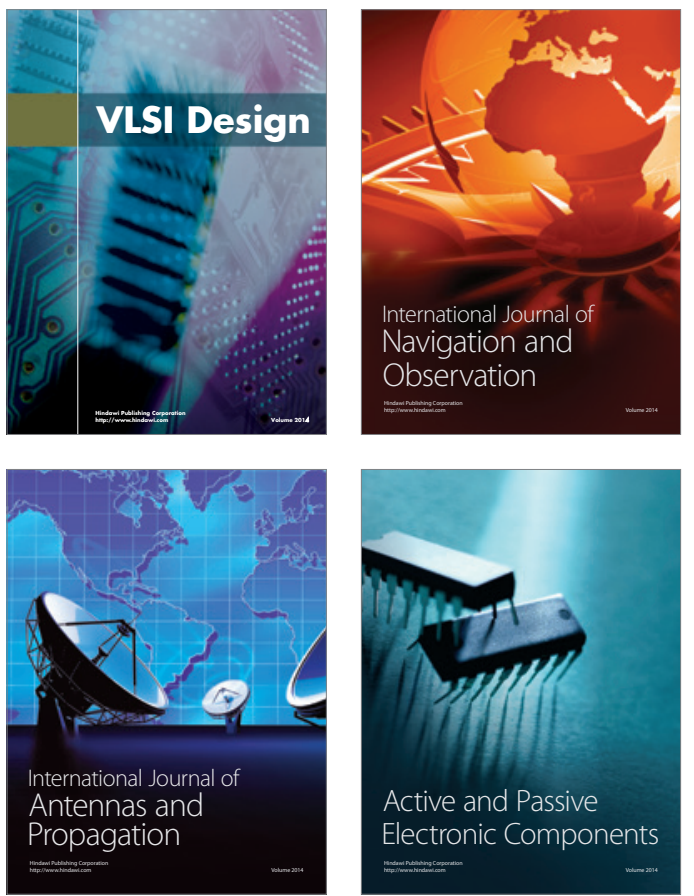
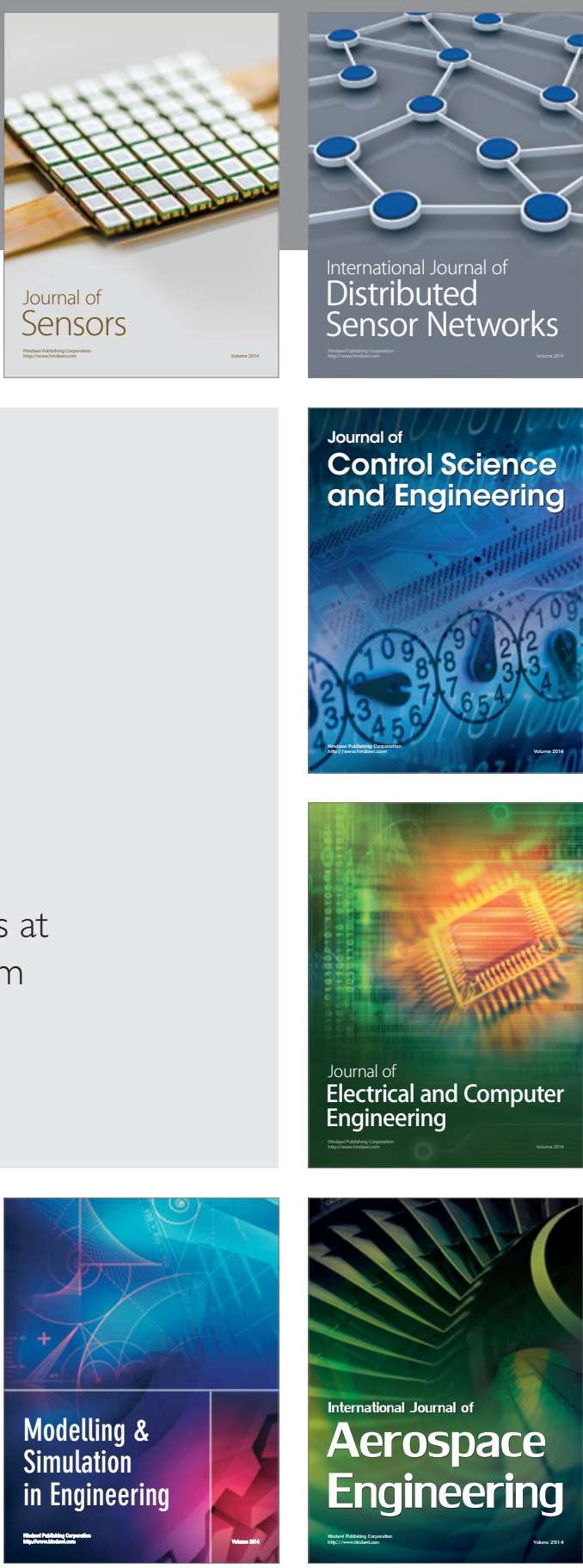

International Journal of

Distributed

Sensor Networks

Journal of

Control Science

and Engineering
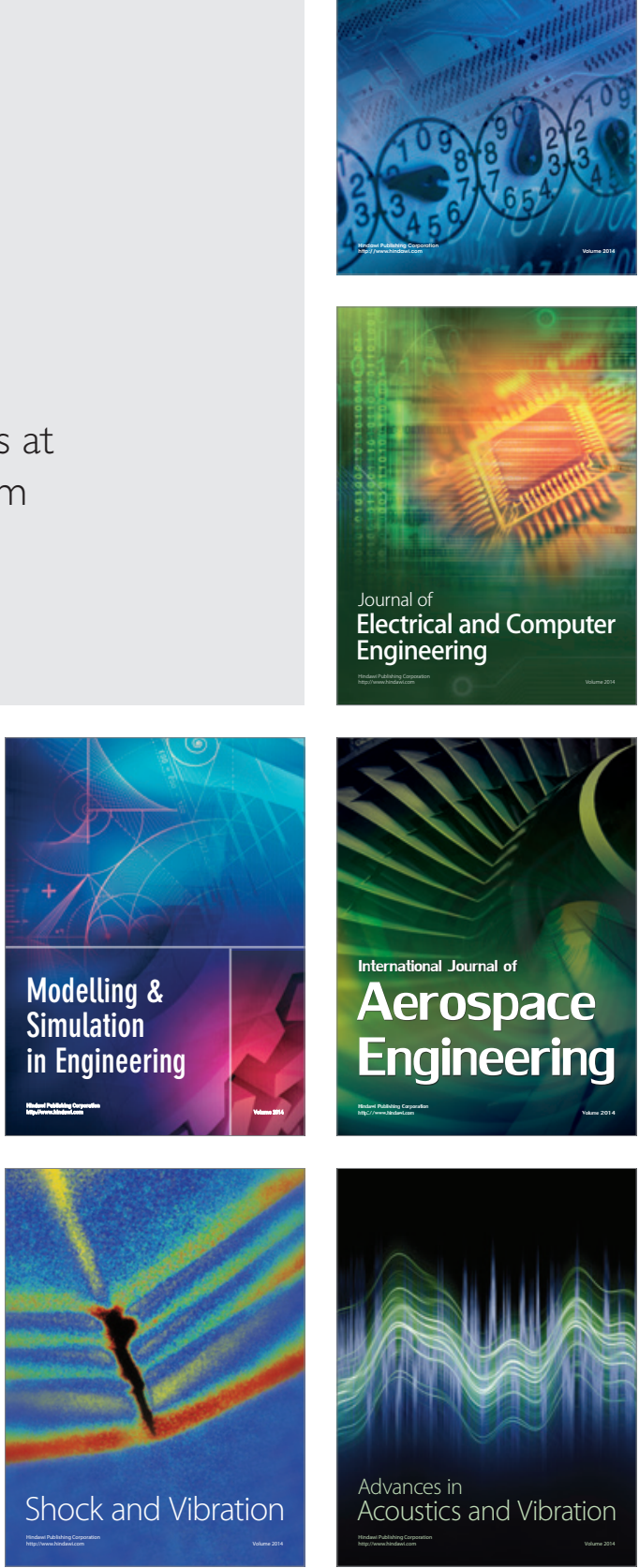